\title{
Testing replication of a 5-SNP set for general cognitive ability in six population samples
}

\author{
Michelle Luciano ${ }^{\star 1,7}$, Penelope A Lind ${ }^{1,2,7}$, Ian J Deary ${ }^{1}$, Antony Payton ${ }^{3}$, \\ Danielle Posthuma $^{4}$, Lee M Butcher ${ }^{5}$, Zoltan Bochdanovits ${ }^{4}$, Lawrence J Whalley ${ }^{6}$, \\ Peter M Visscher ${ }^{2}$, Sarah E Harris ${ }^{1}$, Tinca JC Polderman ${ }^{4}$, Oliver SP Davis ${ }^{5}$, \\ Margaret J Wright ${ }^{2}$, John M Starr ${ }^{1}$, Eco JC de Geus ${ }^{4}$, Timothy C Bates ${ }^{1}$, \\ Grant W Montgomery ${ }^{2}$, Dorret I Boomsma ${ }^{4}$, Nicholas G Martin ${ }^{2}$ and Robert Plomin ${ }^{5}$
}

\footnotetext{
${ }^{1}$ Department of Psychology, MRC Centre for Cognitive Ageing and Cognitive Epidemiology, University of Edinburgh, Edinburgh, UK; ${ }^{2}$ Genetic Epidemiology, Queensland Institute of Medical Research, Brisbane, Queensland, Australia; ${ }^{3}$ School of Medicine, The University of Manchester, Manchester, UK; ${ }^{4}$ Biological Psychology, Vrije Universiteit of Amsterdam, Amsterdam, The Netherlands; ${ }^{5}$ King's College London, Institute of Psychiatry, London, UK; ${ }^{6}$ Mental Health, University of Aberdeen, Aberdeen, UK
}

A 5-single nucleotide polymorphism (SNP) set has been associated with general cognitive ability in 5000 7-year-old children from the Twins Early Development Study (TEDS). Four of these SNPs were identified through a $10 \mathrm{~K}$ microarray analysis and one was identified through a targeted analysis of brain-expressed genes. The present study tested this association with general cognitive ability in six population samples of varying size and age from Australia, the UK (Scotland and England) and the Netherlands. Results from the largest sample $(N=1310)$ approached significance $(P=0.06)$ in the direction of the original finding, but results from the other samples $(N=205-758)$ were mixed. A meta-analysis of the results - allowing for effect size heterogeneity between samples - yielded a non-significant correlation $(r=-0.01, P=0.57)$, indicating that this SNP set was not associated with general cognitive ability in the populations studied. European Journal of Human Genetics (2008) 16, 1388-1395; doi:10.1038/ejhg.2008.100; published online 21 May 2008

Keywords: SNP set; cognitive ability; replication

\section{Introduction}

Cognitive abilities are partly heritable and findings generally show an increase in the influence of genes on mental abilities across the lifespan; heritability estimates of around 0.30 in childhood increase to 0.80 in old age. ${ }^{1,2}$ Molecular genetic studies of cognitive abilities report genetic

${ }^{*}$ Correspondence: Dr M Luciano, Department of Psychology, MRC Centre for Cognitive Ageing and Cognitive Epidemiology, University of Edinburgh, 7 George Square, Edinburgh, Lothian EH8 9JZ, UK.

Tel: + 44131651 5040; Fax: + 44131651 1771;

E-mail: michelle.luciano@ed.ac.uk

${ }^{7}$ The authors wish it to be known that, in their opinion, the first $\mathbf{2}$ authors should be regarded as joint First Authors

Received 30 October 2007; revised 11 March 2008; accepted 17 April 2008; published online 21 May 2008 associations with specific cognitive abilities ${ }^{3,4}$ and with general cognitive ability. ${ }^{5,6-8}$ General cognitive ability captures the covariation between diverse cognitive abilities, and it has been proposed that this covariation reflects underlying 'generalist genes', affecting many cognitive abilities. ${ }^{9}$ Using an approach based on single nucleotide polymorphism (SNP) microarrays ( $\sim 11000$ SNPs) and DNA pooling, Butcher et al, ${ }^{10}$ reported an association between four SNPs and general cognitive ability $(g)$ in 6154 children aged 7 years. These associations were confirmed by individual genotyping and explained $0.76 \%$ of variance in $g$ when they were scored in the direction of a positive association with $g$ and summed to form a composite score. The minor allele of each SNP conferred the greater risk for low $g$ scores. Both dominance and epistasis effects were 
shown not to contribute considerably to the prediction of $g$, and therefore the composite measure was formed assuming an additive model (ie, using scores of 0, 1 and 2 for respective A1A1, A1A2 and A2A2 genotypes).

An SNP previously identified from a scan of 432 brainexpressed polymorphisms ${ }^{6}$ was later added to this set to form a 5-SNP set explaining $0.86 \%$ of variance in $g$ in a largely overlapping sample $(P<0.001)$; this SNP set was slightly more strongly correlated with verbal $\left(r^{2}=0.097\right.$, $P<0.001)$ than non-verbal ability $\left(r^{2}=0.048, P=0.001\right)$. Furthermore, there was evidence that the SNP set explained $17 \%$ of the stable genetic variation between $g$ at age 2 (assessed by a parentally administered cognitive test) and 7 (measured by a telephone administered form of the WISCIII-UK) years. So far, association of this SNP set has been tested only in the Twins Early Development Study (TEDS) sample, although longitudinally. Our aim therefore was to replicate this SNP set association with cognitive ability in six independent samples from Australia, the Netherlands, Scotland and England. The samples were of varying age, measured with diverse cognitive abilities tests and we have focused on general cognitive ability to attain the most similar and reliable cognitive index across cohorts. The 5-SNP set includes: rs1136141, located in heat-shock cognate protein 8 gene (HSPA8) on 11q; rs991684 on chromosome 2, near two known genes and perhaps is itself in a gene; rs726523 (chromosome 18), rs4128492 (chromosome 6) and rs2382591 (chromosome 7), not located in known genes.

\section{Materials and methods \\ Samples}

Australian cohort Twins and their non-twin siblings were initially recruited as part of ongoing studies of melanoma risk factors and cognition. ${ }^{11,12}$ The sample included 1310 individuals (49.2\% male) from 641 families (248 monozygotic [MZ] families with an additional 64 nontwin siblings, 393 dizygotic [DZ] with 111 non-twin siblings). Participants ranged in age from 15 to 22 years (mean age: $16.2 \pm 0.4$ for twins; $17.4 \pm 1.13$ for siblings), with the majority of the sample being Caucasian, predominantly Anglo-Celtic. Written informed consent was obtained from each participant and their parent/guardian (if $<18$ years) before testing.

Dutch cohorts (children, adult) The Dutch children cohort was part of an ongoing study on the genetics of attention, ${ }^{2}$ who were born between 1990 and 1992 and measured on IQ at ages 5 and 12 years. There were 379 individuals (179 males) from 166 families with phenotype and genotype data. At 5 years, $85 \mathrm{MZ}$ and $73 \mathrm{DZ}$ twin pairs participated (mean age of 5.8 years \pm 0.1 ), while at 12 years an additional $5 \mathrm{DZ}$ twins pairs and 48 non-twin siblings of twins participated. On second assessment twins were aged
$12.4 \pm 0.16$ years, with younger siblings being on an average 9.6 \pm 0.71 and older siblings being $14.69 \pm 0.60$. Before the assessment, the parents provided signed informed consent, including a voluntary agreement to provide buccal swabs for DNA isolation and genotyping. The Dutch adult cohort was part of an ongoing study on the genetics of brain function ${ }^{13}$ and consisted of 361 subjects (168 males) from 174 families. There were $42 \mathrm{MZ}$ pairs, $61 \mathrm{DZ}$ pairs, $1 \mathrm{DZ}$ triplet and 152 siblings. Mean age at the time of testing was $36.4 \pm 12.4$ years. Participants voluntarily agreed to donate blood for DNA study.

Scottish cohorts (Lothian, Aberdeen) Recruitment of Lothian Birth Cohort 1921 (LBC1921) has been described previously. ${ }^{14}$ Briefly, the LBC1921 consisted of 526 subjects (219 males) with both phenotype and genotype data; they had participated in the Scottish Mental Survey of 1932 at the age of 11 years, and were retested between 1999 and 2001 at a mean age of 79. All participants lived independently in the community. For this study, inclusion criteria were no history of dementia and a Mini-Mental State Examination (MMSE) score of 24 or greater. Recruitment of the Aberdeen Birth Cohort 1936 (ABC1936) has been described previously, ${ }^{14}$ genotype data were available for 205 subjects (109 males) who took the Scottish Mental Survey of 1947 at the age of 11 years, and were retested at age 64. All participants lived independently in the community. Inclusion criteria were the same as for LBC1921. Ethics permission was granted from the Lothian and Grampian Research Ethics Committees, respectively, for the LBC1921 and the ABC1936 and all subjects gave written, informed consent to the study.

English cohort The 758 Caucasian volunteers involved in this study form part of the Dyne Steele DNA bank for cognitive genetic studies and comprise 234 males and 524 females. On entry to the study, the age range was 50-85 years and the mean age was 63 years. Cognitive tests were given at five yearly intervals up to 15 years later. At the beginning of the study, all volunteers achieved the maximum score on the MMSE, and at the time of venesection (11-15 years later), cognitive tests indicated no sign of dementia. Recruitment and sample composition details are described elsewhere. ${ }^{15}$ Volunteers gave written consent for the use of their DNA.

TEDS Caucasian participants without serious medical or perinatal problems were drawn from a subsample of 7410 twins from the TEDS ${ }^{16}$ who took part in a study of pooled DNA genotyped on microarrays. ${ }^{10}$ Of these, 6154 had general cognitive ability data and 4836 of these had complete genotyping data for the 5-SNPs. Of the 4836 individuals, 1516 were MZ pairs (421 girl pairs, 674 boy), 17 were unpaired MZ twins (7 girls, 10 boys), 1816 were same-sex DZ pairs (470 girl pairs, 438 boy), 1144 were in 
opposite-sex DZ pairs and 343 were unpaired DZ twins (173 girls, 170 boys).

\section{Measures \\ Australian cohort IQ data were collected in the labora- tory using the shortened version of the Multidimensional Aptitude Battery: three verbal subtests, (information, arithmetic, vocabulary) and two performance subtests (spatial, object assembly). Scaled scores for Full IQ were calculated following the manual instructions.}

Dutch cohorts For the Dutch children cohort at age 5, IQ was assessed with a shortened version of the RAKIT, a Dutch intelligence test: ${ }^{17}$ exclusion, discs, hidden figures, verbal meaning, learning names and idea production. At age 12, IQ was assessed with the Dutch adaptation of the WISC- $\mathrm{R}^{18}$ using a shortened version (similarities, vocabulary, arithmetic, digit span, block design and object assembly). For the Dutch adult cohort, cognitive ability was assessed with the Dutch adaptation of the WAIS-IIR. ${ }^{19}$

Scottish cohorts All LBC1921 and ABC1936 subjects took a version of the Moray House Test, No. 2 $\left(\mathrm{MHT}^{20,21}\right)$, a general mental ability test at 11 years, in the Scottish Mental Surveys of 1932 and 1947, respectively. The test was previously described in detail. ${ }^{14}$ LBC1921 repeated the same test at about 79 years, along with the Raven's Standard Progressive Matrices a measure of non-verbal reasoning, ${ }^{22}$ the Wechsler logical memory test, a test of verbal fluency and the National Adult Reading Test (NART), which provides an estimate of prior IQ. ${ }^{23,24}$ The ABC1936 was retested at about 64 years on the Raven and the NART. The MHT scores were used to index $g$ at age 11 in both cohorts. In the LBC1921 at 79 years, regression factor scores of the first unrotated principal component extracted from the five tests were taken as the measure of $g$ derived in SPSS, ${ }^{25}$ while in the ABC1936 at 64 years, the two measures were standardised and summed to attain a global cognitive ability measure.

English cohort Tests of fluid intelligence comprised the Heim intelligence tests parts one and two. ${ }^{26}$ Processing speed was assessed using the random letters test. A series of memory tests measured semantic memory, immediate verbal recall and delayed verbal recall. A general factor was extracted from these tests and regression factor scores used as a measure of $g$ (as previously described). Measures of full scale IQ and $g$ have been shown to be strongly correlated $(\sim 0.90)$ in previous research. ${ }^{27}$ As this was an ageing sample, data at the first time point only were investigated to avoid confounding of differential cognitive decline due to dementia.

TEDS Two verbal subtests - Vocabulary, Similarities WISC-III-UK, ${ }^{28}$ - and two non-verbal subtests - Picture
Completion (WISC-III-UK), Conceptual Grouping McCarthy Scales of Children's Abilities, ${ }^{29}$ were administered via telephone. ${ }^{30}$ Subtest scores were standardised on the full sample and summed to create $g$ scale scores.

\section{Genotyping}

Australian cohort Assays for the five SNPs (rs4128492, rs2382591, rs726523, rs1136141, rs991684) were designed using MassARRAY Assay Design software (version 3.0; Sequenom Inc., San Diego, CA) and typed using iPLEX ${ }^{\mathrm{TM}}$ chemistry on a Compact MALDI-TOF Mass Spectrometer (Sequenom Inc., San Diego, CA). Forward and reverse PCR primers and a primer extension probes were purchased from Bioneer Corporation (Daejeon, Korea). The iPLEX reaction products were desalted by diluting samples with $18 \mu \mathrm{l}$ of water and $3 \mu \mathrm{l}$ of resin to optimise mass spectrometric analysis and then spotted on a SpectroChip (Sequenom), processed and analysed by the MassARRAY Workstation software (version 3.3; Sequenom). Assay quality and genotype calls were assessed in the SpectroTYPER software (version 3.3; Sequenom). The genotypes for one assay (rs4128492) could not be accurately usercalled and was excluded from analysis.

Dutch cohort Genotyping was performed blind to familial status and phenotypic data. Both MZ twins of a pair were included, serving as additional quality control on genotyping. Genotyping was performed using fluorogenic probes in the high-throughput $5^{\prime}$-nuclease assay (TaqMan, PE Applied Biosystems, Foster city, CA), which combines polymerase chain reaction amplification and detection into a single step. The assay requires two allele-specific probes, which labelled two alleles with different fluorescent reporter dyes for discrimination. Following allelespecific hybridisation, the detection probe is cleaved during each amplification cycle by the 5'-exonuclease activity of Taq DNA polymerase if the probe's target sequence is present. No TaqMan assay for rs1136141 could be designed due to the presence of a nearby SNP, and the TaqMan assay for rs4128492 failed.

Scottish and English cohorts The Sequenom Mass ArrayTM (Sequenom Inc., Germany) was used for genotyping SNPs in all cohorts, with the exception of rs4128492 in the Scottish cohorts. PCR iPLEX oligoprimers were designed and optimised using MassARRAY Assay Design software v.3.0. PCR reaction mix $(5 \mu \mathrm{l})$ consisted of $2.92 \mu \mathrm{l}$ deionized water, $0.625 \mu \mathrm{l} 10 \times$ HotStar Taq PCR Buffer (15 mM MgCl 2 ), $0.325 \mu \mathrm{l} 25 \mathrm{mM} \mathrm{MgCl}_{2}, 0.1 \mu \mathrm{l}$ dNTPs $25 \mathrm{~mm}$ each, $0.03 \mu \mathrm{l}$ HotStar Taq Polymerase (QIAgen) (5 U/ $\mu \mathrm{l})$, $1.0 \mu \mathrm{l}$ Forward and Reverse Primer Mix (500 nm each) and $25 \mathrm{ng}$ of genomic DNA. PCR cycles: $95^{\circ} \mathrm{C} 15 \mathrm{~min}$ followed by $95^{\circ} \mathrm{C} 20 \mathrm{~s}, 56^{\circ} \mathrm{C} 30 \mathrm{~s}, 72^{\circ} \mathrm{C} 60 \mathrm{~s}$ (35 cycles) and finally $72^{\circ} \mathrm{C}$ for $3 \mathrm{~min}$. In the Scottish cohorts, rs4128492 was genotyped at the Wellcome Trust Clinical Research Facility 
Genetics Core, Western General Hospital, Edinburgh (http://www. wtcrf.ed.ac.uk/genetics/index.htm) using TaqMan technology (Applied Biosystems).

TEDS Individual genotyping and allele calling for the TEDS cohort was outsourced to Kbiosciences, UK, who use a mixture of competitive allele specific PCR (KASPar) and TaqMan genotyping assays (http://www.kbioscience.co. uk/). Kbioscience consider an assay successful if four quality control criteria are met: (1) three distinct clusters, (2) water controls must be negative, (3) number of genotypes callable must be $>90 \%$ and (4) minor allele frequency should be greater than $2 \%$.

Genotype error checking, including Mendelian inconsistencies, and tests of Hardy-Weinberg equilibrium were performed in MERLIN (http://www.sph.umich.edu/csg/ abecasis/Merlin, ${ }^{31}$ ) and Sib-pair (http://www2.qimr.edu.au/ davidD/sib-pair.html, ${ }^{32}$ ).

\section{Association analyses}

To calculate the SNP set score, the genotypes for each of the SNPs was recoded so that the decreaser allele homozygote was assigned a value of 0 , the heterozygote assumed a value of 1 and the increaser allele homozygote was assigned a value of 2 . These values were then summed across SNPs to derive an SNP set score. Association analyses were performed in $\mathrm{Mx}^{33}$ which uses a maximum likelihood procedure to estimate parameters in a means model (to test the association effect). The means model included the fixed effects of relevant covariates in addition to a linear regression term for the SNP set effect. As SNPs were scored in the direction of the increaser allele, a positive correlation between $g$ and the SNP set was expected irrespective of the differing number of SNPs contained in each set between cohorts (this varied from three to five).

In the Australian, Dutch and TEDS cohorts, the twin relationship was modelled in the covariance structure, separately for MZ and DZ twins, to account for the nonindependence of siblings within a family. For the Dutch child cohort, the covariance between time points was further modelled to account for the longitudinal nature of the data. Likewise, the covariance between time points was modelled in the Scottish cohorts. To test the significance of the SNP set effect, the linear regression term for this measure was fixed to zero and the resulting model was compared to the model in which the regression term was estimated, using the likelihood ratio $\chi^{2}$-test. The difference in -2-log likelihood between these models is distributed as a $\chi^{2}$ with the degrees of freedom equal to the difference in degrees of freedom between the two models (ie, 1 for the SNP set effect). The power to detect an effect size of $0.8 \%$ (given an average marker allele frequency of 0.35 ) was $88 \%$ in the Australian sample, 70\% in the English sample, 54\% in the LBC1921, 40\% in the Dutch adults, 34\% in the Dutch children and $25 \%$ in the ABC1936. The power to detect the smaller, individual SNP effects was much lower. A meta-analysis of the results excluding the TEDS sample was performed using the Comprehensive Meta-analysis package. $^{34}$ The correlation was used as the effect size measure and a random effects model was specified to account for systematic differences between the sampled populations.

As the original finding was reported in children, we analysed the SNP set effect separately for young (Dutch, LBC1921, ABC1936 children, Australian adolescents) and old (Dutch, LBC1921, ABC1936, English adults) cohorts. We further estimated the SNP set association with $g$ separately for males and females because of a report that the $g$ SNP set association is significantly stronger in males than females at age $10 .^{35}$

\begin{abstract}
Results
Genotype screening showed that the population was not in Hardy-Weinberg equilibrium $(P=0.005)$ at SNP rs1136141 in the Australian sample, with heterozygote genotypes underrepresented. No other deviations from Hardy-Weinberg $(P<0.01)$ were observed in any of the other samples. With such a large number of independent tests performed (3-5 SNPs in six samples), it is probable that the deviation in the Australian sample was a false positive, so this SNP was retained for further analysis (note that analyses excluding this SNP from the SNP set score produced consistent results - data not shown). Allele frequencies for the five SNPs were consistent with reported frequency data in Caucasians, and did not differ between cohorts (see Table 1). The frequency distribution of the SNP set score
\end{abstract}

Table 1 Minor allele frequencies for the 5 SNPs in each of the six replicate cohorts and in the original TEDS cohort

\begin{tabular}{|c|c|c|c|c|c|c|c|c|c|}
\hline & \multicolumn{2}{|c|}{$\mathrm{Ch}$} & Australian & English & Scottish ABC1936 & Scottish LBC1921 & Dutch children & Dutch adults & TEDS \\
\hline rs991684 & 2 & $A^{*} G$ & 0.30 & 0.29 & 0.30 & 0.28 & 0.31 & 0.29 & 0.28 \\
\hline rs4128492 & 6 & $A G^{*}$ & - & 0.24 & 0.22 & 0.24 & - & - & 0.24 \\
\hline rs2382591 & 7 & $A^{*} C$ & 0.16 & 0.14 & 0.17 & 0.15 & 0.15 & 0.15 & 0.16 \\
\hline rs726523 & 18 & $G A^{*}$ & 0.23 & 0.24 & 0.24 & 0.24 & 0.22 & 0.22 & 0.23 \\
\hline rs1136141 & 11 & $G A^{*}$ & 0.14 & 0.13 & 0.14 & 0.15 & - & - & 0.14 \\
\hline
\end{tabular}

Note: *represents minor allele; — represents SNPs that failed genotyping procedures. 
Table 2 SNP set associations with $g$ in TEDS, the six replicate cohorts, and the meta-analysis of all cohorts excluding TEDS

\begin{tabular}{|c|c|c|c|c|c|c|c|}
\hline Cohort & $N^{a}$ & SNP correlation & $95 \% \mathrm{Cl}$ & P-value & \multicolumn{3}{|c|}{ SNP correlation and $95 \% \mathrm{Cls}$} \\
\hline TEDS & 4836 & 0.08 & $(0.05,0.11)$ & 0.000 & & & \\
\hline Dutch 5 years & 369 & 0.05 & $(-0.07,0.17)$ & 0.389 & & & \\
\hline Dutch 12 years & 379 & -0.04 & $(-0.14,0.05)$ & 0.141 & & & \\
\hline Australian & 1310 & 0.06 & $(0.00,0.12)$ & 0.063 & & & \\
\hline LBC1921 11 years & 526 & -0.11 & $(-0.02,-0.20)$ & 0.009 & & & \\
\hline LBC1921 79 years & 526 & -0.07 & $(-0.16,0.02)$ & 0.074 & & & \\
\hline$A B C 193611$ years & 205 & 0.05 & $(-0.05,0.14)$ & 0.330 & & & \\
\hline$A B C 193664$ years & 205 & 0.10 & $(0.01,0.20)$ & 0.022 & & & \\
\hline English & 758 & -0.07 & $(-0.15,0.00)$ & 0.053 & & & \\
\hline Dutch adult & 361 & -0.07 & $(-0.19,0.05)$ & 0.225 & & & \\
\hline Meta-analysis & 3539 & -0.01 & $(-0.06,0.03)$ & 0.570 & -1.0 & -0.5 & 1.0 \\
\hline
\end{tabular}

${ }^{\mathrm{a}} \mathrm{N}$ contains $\mathrm{MZ}$ pairs for TEDS, Dutch and Australian cohorts.

was consistent with the distribution reported in the TEDS sample, displaying a slight negative skew.

Significant mean effects were shown for sex, months of schooling, and sibling status (twin versus non-twin sibling) in the Australian cohort, with males scoring higher than females, a greater amount of schooling relating to better test performance and non-twin siblings scoring higher than twins. In the Dutch child cohort, there were no sex differences at age 5 , although boys scored higher than girls at age 12. In the Dutch adult cohort, men scored higher than women and age was negatively correlated with $g$. In the Scottish cohorts, age in days was positively associated with $g$ at 11 years in the ABC1936 and negatively associated with $g$ at 79 years in the LBC1921. Age and socio-economic status (SES) were significant in the English cohort, with younger participants performing better and higher SES relating to increased cognitive test performance. The tests of association were modelled with inclusion of the relevant covariates or performed using covariateadjusted scores (standardised residuals).

The SNP set correlations and their 95\% confidence intervals are shown in Table 2, separately for each cohort, and for the meta-analysis excluding TEDS. The largest sample (Australian) showed a marginally significant $(P=0.06)$ effect in the expected direction; however, the results for the other studies were mixed - half showed positive results and the other half showed negative results. A significant association in the same direction as the initial TEDS finding was observed in the ABC1936 at 64 years $(r=0.10, P=0.02)$; while in the LBC1921 at 11 years a significant association $(r=-0.11, P=0.01)$ in the reverse direction was found. Positive and negative correlations between $g$ and SNP set score occurred with roughly equal frequency. A homogeneity test (using the $\chi^{2}$ - statistic) of the effect size between samples was significant $(P<0.001)$ indicating that the effect size differed between cohorts. The meta-analysis of the results that allowed for systematic and unsystematic differences in effect size between cohorts showed a non-significant correlation of $-0.01(P=0.57)$. Despite reduced power, the effect of individual SNPs was tested within each cohort. No significant association was found for markers rs726523 and rs1136141 in any of the cohorts. For rs991684, a significant association $(r=0.13$, $P=0.007)$ in the hypothesised direction was found in the ABC1936 cohort at 64 years. For rs4128492 and rs2382591, respective correlations of $-0.10(P=0.007)$ and -0.09 $(P=0.16)$ were found in the English cohort, with the direction of the effect being inconsistent with the original finding in the TEDS sample.

Age effects were estimated by fixing the SNP set regression coefficient equal within young replicate cohorts (Australian, Dutch children, LBC1921 and ABC1936 at 11 
years) and old replicate cohorts (English, Dutch adults, LBC1921 and ABC1936 adults). The test of the homogeneity of the SNP set effect within young and old cohorts was significant $(P<0.01)$. Nevertheless, the test of association in both young and old cohorts was not significant $(P=1)$. The sex analyses of the SNP set showed negative association $(r=-0.03)$ for males in the ABC1936 $(P=0.01)$ at 11 years and positive association $(r=0.04)$ for females in the Dutch children at age $5(P=0.04)$. Positive correlations were found in the ABC1936 $(P=0.04)$ at 64 years in females $(r=0.13)$ and males $(r=0.07)$.

\section{Discussion}

This is the first study to attempt replication of a 5-SNP set influencing general cognitive ability. ${ }^{36}$ These SNPs were originally identified through DNA pooling comparing low and high groups in microarray analysis selected from a large sample of children and confirmed using individual genotyping in the entire sample. Our study showed that in six population-based samples, the SNP set effect (in the expected direction) was replicated in one cohort. In a further cohort significant association was observed, but the SNP set effect was in the reverse direction to that expected. A meta-analysis of these results did not support an association between the SNP set and $g$.

Empirical tests of the equality of the SNP set $\beta$-coefficient showed significant heterogeneity in the effect size between the cohorts. Our more focussed analyses showed that neither sex nor age could explain these differences in effect size as heterogeneity was also observed within samples of the same sex and of similar age. The SNP set was not related to $g$ in either young or old pooled samples nor was an enhanced effect of the SNP set effect observed in males (positive correlations were found in female and male groups or in females only). The original SNP set effect was shown in the TEDS cohort at ages 2,4 and 7 . In the cohort most comparable to the TEDS sample, the Dutch cohort measured at age 5, the correlation was positive $(r=0.05)$, though not significant. Given the low power of this sample, it remains possible that the SNP set effect is important in early childhood. There is some evidence for different genes affecting IQ in the TEDS sample during childhood although most genetic effects are stable from year to year; ${ }^{37,38}$ research in Dutch samples supports a largely stable influence of genes on IQ at ages 5 and 12 (with amplification of genetic effects during development). ${ }^{2,39}$

Other explanations for the variation in the size of the SNP set effect between the cohorts were sought. The use of diverse cognitive measures might have been a factor, especially if the SNP set influences variation on some measures more than others. The original finding in TEDS reported a stronger relationship with verbal than non- verbal ability. In our cohorts, the $g$ factor was extracted using different test batteries with some $g$ factors therefore tapping more verbal ability variance than others; it is unclear to what extent the $g$ factor between studies is measuring the same underlying processes. Another factor contributing to variability of the effect might be differences in the SNP set size tested (due to failed genotyping) across samples, with restriction in range of the SNP set size leading to attenuated correlations in the samples with missing genotype data.

While the finding of negative associations was unexpected, this 'flip-flop' association is not uncommon in replication studies of $\mathrm{SNPs}^{40}$ and may stem from epistatic effects in which other interacting alleles at unobserved loci have different frequencies across the different populations. It must be noted that a higher number of tests were significant (9) than expected by chance (4), with five of these in the predicted direction; furthermore, 5 out of 9 tests for the SNP set analyses showed a $P$-value less than 0.10. It is possible then that the SNP set has predictive validity when coupled with other unidentified SNPs contributing to $g$.

The SNP set approach appears as a reasonable method to test for the association of multiple gene effects on a trait, reducing problems associated with multiple testing of individual genes and with detecting very small effect sizes of individual SNPs. However, the approach assumes that individual SNPs in a set contribute equal amounts of variance to a trait. When allele frequencies differ between SNPs, those SNPs with greater heterozygosity will carry a larger influence on the SNP set score. In this study, allele frequencies of the SNPs were similar, with little expected bias in the results. For future analyses of SNP set scores, it may be useful to weigh the SNPs by their effect size.

The present study highlights the importance of replication studies, and what will be an increasing need for them in light of the burgeoning era of genome-wide association (GWA) studies. This is especially so for two reasons. First, given that GWA studies now incorporate hundreds of thousands of SNPs rather than the 10000 used in the original TEDS study; while these studies will have more success in finding true genetic variants they will also detect a larger number of associations due to chance. Second, although GWA studies can reliably detect large effect sizes as in the early exciting report for age-related macular degeneration, ${ }^{41}$ the largest effects in recent GWA studies such as the Wellcome Trust Case Control Consortium ${ }^{42}$ are very small, which will be difficult to replicate. Although the TEDS two-stage design had considerable power to detect small effects, the effect sizes of the associations that were detected were smaller than warranted by the power of the design. In other words, although GWA studies will easily detect any large effects, identifying the 'best of the rest' will be challenging. Demands for power will be just as daunting for replication studies. An innovation of the 
TEDS study was to aggregate candidate SNPs of small effect size together in an additive SNP set which can be tested for replication with greater power; however, the SNP set is only as good as its constituent SNPs.

In our meta-analysis, which allowed heterogeneity of the SNP set effect between samples, the SNP set was not correlated with $g$. While the SNP set is not related to general cognitive ability in the cohorts we tested, the ideal replication study would be the one conducted in a large sample of children more closely matched in age to that of the TEDS and using the same cognitive measures.

\section{Acknowledgements}

We thank the participants and their families for their cooperation. Australian data collection was funded by ARC grants (A79600334, A79906588, A79801419, DP0212016, DP0343921) and the Human Frontiers of Science Program (HFSP; Grant rg0154/1998-B). Dutch data collection was supported by the Universitair Stimulerings Fonds (Grant 96/22), the HFSP (Grant rg0154/1998-B), and the Netherlands Organization for Scientific Research (NWO)/ Centre for Medical Systems Biology (CMSB) (Grant NWO/SPI 56-464-14192 \& 904-57-94 and NWO/MaGW VIDI-016-065-318). The Lothian Birth Cohort 1921 is supported by the UK's Biotechnology and Biological Sciences Research Council. The Aberdeen Birth Cohort 1936 is supported by the Wellcome Trust. IJD is the recipient of a Royal Society-Wolfson Research Merit Award. Blood collection and DNA extraction for the Dyne Steele cohort was partly funded by Research into Ageing. The Twins Early Development Study is supported by the UK Medical Research Council (G0500079) and the Wellcome Trust (GR75492).

\section{References}

1 McClearn GE, Johansson B, Berg S et al: Substantial genetic influence on cognitive abilities in twins 80 or more years old. Science 1997; 276: 1560-1563.

2 Polderman TJ, Gosso MF, Posthuma D et al: A longitudinal twin study on IQ, executive functioning, and attention problems during childhood and early adolescence. Acta Neurol Belg 2006; 106: 191-207.

3 Papassotiropoulos A, Stephan DA, Huentelman MJ et al: Common Kibra alleles are associated with human memory performance. Science 2006; 314: 475-478.

4 Schaper K, Kolsch H, Popp J, Wagner M, Jessen F: KIBRA gene variants are associated with episodic memory in healthy elderly. Neurobiol Aging 2007 (in press).

5 Plomin R, Turic DM, Hill L et al: A functional polymorphism in the succinate-semialdehyde dehydrogenase (aldehyde dehydrogenase 5 family, member A1) gene is associated with cognitive ability. Mol Psychiatry 2004; 9: 582-586.

6 Butcher LM, Meaburn E, Dale PS et al: Association analysis of mild mental impairment using DNA pooling to screen 432 brainexpressed single-nucleotide polymorphisms. Mol Psychiatry 2005; 10: $384-392$.

7 Gosso MF, de Geus EJ, van Belzen MJ et al: The SNAP-25 gene is associated with cognitive ability: evidence from a family-based study in two independent Dutch cohorts. Mol Psychiatry 2006; 11: 878-886.

8 Gosso MF, van Belzen M, de Geus EJ et al: Association between the CHRM2 gene and intelligence in a sample of 304 Dutch families. Genes Brain Behav 2006; 5: 577-584.
9 Plomin R, Kovas Y: Generalist genes and learning disabilities. Psychol Bull 2005; 131: 592-617.

10 Butcher LM, Meaburn E, Knight J et al: SNPs, microarrays and pooled DNA: identification of four loci associated with mild mental impairment in a sample of 6000 children. Hum Mol Genet 2005; 14: 1315-1325.

11 McGregor B, Pfitzner J, Zhu G et al: Genetic and environmental contributions to size, color, shape, and other characteristics of melanocytic naevi in a sample of adolescent twins. Genet Epidemiol 1999; 16: 40-53.

12 Wright M, De Geus E, Ando J et al: Genetics of cognition: outline of a collaborative twin study. Twin Res 2001; 4: 48-56.

13 Posthuma D, de Geus EJ, Mulder EJ, Smit DJ, Boomsma DI, Stam CJ: Genetic components of functional connectivity in the brain: the heritability of synchronization likelihood. Hum Brain Mapp 2005; 26: 191-198.

14 Deary IJ, Whiteman MC, Starr JM, Whalley LJ, Fox HC: The impact of childhood intelligence on later life: following up the Scottish mental surveys of 1932 and 1947. J Pers Soc Psychol 2004; 86: 130-147.

15 Rabbitt P, Diggle P, Holland F, McInnes L: Practice and drop-out effects during a 17-year longitudinal study of cognitive aging. J Gerontol B Psychol Sci Soc Sci 2004; 59: P84-P97.

16 Oliver B, Plomin R: Twins' Early Development Study (TEDS): a multivariate, longitudinal genetic investigation of language, cognition and behavior problems from childhood through adolescence. Twin Res Hum Genet 2007; 10: 96-105.

17 Bleichrodt N, Drenth PJD, Zaal JN, Resing WCM: Revisie amsterdamse Kinder Intelligentie Test [Revised Amsterdam Child Intelligence Test]. Lisse, The Netherlands: Swets and Zeitlinger BV, 1984.

18 Van Haasen PP, De Bruyn EEJ, Pijl YJ et al: Wechsler Intelligence Scale for Children-Revised. Dutch Version. Lisse, The Netherlands: Swets and Zeitlinger BV, 1986.

19 Wechsler D: WAIS-III Weschler Adult Intelligence Scale. San Antonio, Texas: Psychological Coorporation, 1997.

20 Scottish Council for Research in Education: The intelligence of Scottish children: A national survey of an age-group, Vol V London: University of London Press, 1933.

21 Deary IJ, Whalley LJ, Lemmon H, Crawford JR, Starr J: The stability of individual differences in mental ability from childhood to old age: follow-up of the 1932 Scottish Mental Survey. Intelligence 2000; 28: 49-55.

22 Raven JC, Court JH, Raven J: Manual for Raven's Progressive Matrices and Vocabulary Scales. London: HK Lewis, 1977.

23 Crawford JR, Deary IJ, Starr J, Whalley LJ: The NART as an index of prior intellectual functioning: a retrospective validity study covering a 66-year interval. Psychol Med 2001; 31: 451-458.

24 Nelson HE: National Adult Reading Test. Berkshire: NFER - Nelson Publishing Company, 1982.

25 SPSS Inc.: SPSS 13.0 for Windows. Chicago, Illinois: SPSS Inc., 2004.

26 Heim A: Intelligence and Personality: Their Assessment and Relationship. Harmondsworth, UK: Penguin, 1970.

27 Jensen AR: The g Factor: The Science of Mental Ability. Westport, CT: Praeger, 1998.

28 Wechsler D: Wechsler Intelligence Scale for Children. London: Psychological Corporation, 1992.

29 McCarthy D: Manual for the McCarthy Scales of Children's Abilities. New York: The Psychological Corporation, 1972.

30 Petrill SA, Rempell J, Oliver B, Plomin R: Testing cognitive abilities by telephone in a sample of 6-8-year olds. Intelligence 2002; 30: 353-360.

31 Abecasis GR, Cherney SS, Cookson WO, Cardon LR: Merlin-rapid analysis of dense genetic maps using sparse gene flow trees. Nat Genet 2002; 30: 97-101.

32 Duffy DL: SIB-PAIR: A Program for Elementary Genetical Analyses 2005, http://www2.qimr.edu.au/davidD/sib-pair.html.

33 Neale MC, Boker SM, Xie G, Maes HH: Mx: Statistical Modeling, VCU Box 900126 Richmond, VA: Department of Psychiatry, 1999. 
34 Borenstein M, Hedges L, Higgins J, Rothstein H: Comprehensive Meta-analysis. Englewood NJ: Biostat, 2005.

35 Arden R, Harlaar N, Plomin R: Sex differences in childhood associations between DNA markers. Journal of Individual Differences, in press.

36 Harlaar N, Butcher LM, Meaburn E, Sham P, Craig IW, Plomin R: A behavioural genomic analysis of DNA markers associated with general cognitive ability in 7-year-olds. J Child Psychol Psychiatry 2005; 46: 1097-1107.

37 Davis OSP, Arden R, Plomin R: $g$ in middle childhood: moderate genetic and shared environmental influence using diverse measures of general cognitive ability at 7,9 and 10 years in a larger population sample of twins. Intelligence 2007; 36: $68-80$.
38 Kovas Y, Haworth CMA, Dale PS, Plomin R: The genetic and environmental origins of learning abilities and disabilities in the early school years. Monogr Soc Res Child Dev 2007; 72: 1-144.

39 Bartels M, Rietveld MJH, van Baal GCM, Boomsma DI: Genetic and environmental influences on the development of intelligence. Behav Genet 2002; 32: 237-249.

40 Lin PI, Vance JM, Pericak-Vance MA, Martin ER: No gene is an island: the flip-flop phenomenon. Am J Hum Genet 2007; 80: 531-538.

41 Klein RJ, Zeiss C, Chew EY et al: Complement factor $\mathrm{H}$ polymorphism in age-related macular degeneration. Science 2005; 308: 385-389.

42 The Wellcome Trust Case Control Consortium: Genome-wide association study of 14000 cases of seven common diseases and 3000 shared controls. Nature 2007; 447: 661-678. 